\title{
Pulse response of a nonlinear layer
}

\author{
Claes M. Hedberga) \\ Blekinge Institute of Technology, 37179 Karlskrona, Sweden
}

Oleg V. Rudenko
Moscow State University, Moscow 119899, Russia and Blekinge Institute of Technology,
37179 Karlskrona, Sweden

(Received 19 December 2000; accepted for publication 15 July 2001)

\begin{abstract}
A simple analytical theory is developed for the description of the non-steady-state response of a thin nonlinear layer, which differs markedly in its linear properties from the surrounding medium. Such a layer can model the behavior of real inhomogeneities like a cloud of gas bubbles in a liquid, a crack or split plane in a solid, or the contact between two slightly tightened rough surfaces. Both weakly nonlinear pulse and harmonic responses are calculated and the general properties of the spectral and temporal structures of the scattered field are discussed. Exact strongly nonlinear solutions are derived for a special type of stress-strain relationship corresponding to the behavior of real condensed media under strong loads. Profiles and spectra shown conform with experimental results. The pulse response on the short $\delta$-pulse shaped incident wave is calculated for arbitrary nonlinear properties of the layer. The possibilities to apply the sets of data on measured characteristics of pulse response in the solution of inverse problems are briefly discussed. (C) 2001 Acoustical Society of America. [DOI: 10.1121/1.1402117]
\end{abstract}

PACS numbers: 43.25.Ba, 43.25.Jh [MFH]

\section{INTRODUCTION}

It is well known that a huge nonlinear acoustic response is obtained from liquids containing air bubbles. ${ }^{1-3}$ The response indicates a magnitude of nonlinearity $\epsilon$ for the gasliquid mixture of up to $10^{3},{ }^{1}$ which far exceeds the nonlinearity of homogeneous fluids. For example, water has $(\epsilon$ $\approx 3.5)$ and air has $(\epsilon=1.2)$. The high sensitivity of nonlinear testing has application in the detection of microbubbles, early in the development of boiling or in the detection of fermentation processes. The medical agent "Albunex" is used for nonlinear ultrasonic imaging of the blood flow; similarly, nitrogen bubbles can be detected in the blood of divers.

The huge increase in nonlinear response of solids containing defects in their structure (cracks, loosely packed grains, pores filled in part by gas and liquid, dislocations, residual stresses, etc.) has found widespread use in the testing of composites, buildings, structural materials, industrial products, and rocks (see, for example, Refs. 5-9).

In the last few years new interesting aspects have sparked the interest in nonlinear nondestructive testing (NNDT). ${ }^{10,11}$ A review of experimental results on material characterization including nonlinear reflection methods, is given in Ref. 12.

Needless to say, NNDT calls for the solution of inverse problem, i.e., the reconstruction of shapes, sizes, or other physical properties of inhomogeneities using measurements of the scattered field. In general, the inverse problem of NNDT can be related to nonlinear diffraction tomography. ${ }^{13}$ However, supplementary a priori information is necessary for efficient solution of the inverse problem, which can result

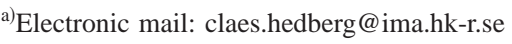

from the solution of direct problems on nonlinear transmission, reflection, or scattering. Unfortunately the set of available direct data leaves something to be desired.

As recent attention has focused on NNDT, extension of the known linear solutions to the corresponding nonlinear problems is a natural path to follow. The problem of normal incidence of a plane wave on a layer is one of the simplest and most important problems in the acoustics of layered media. ${ }^{14}$ It attracts considerable interest for two reasons. First, it is rather simple, and one can look forward to obtaining the solution, which must be in analytical form. Second, the layer can serve as a model of a real nonlinear inclusion such as a cloud of bubbles in a liquid, or a region inside a solid with a high content of defects, or a resonant cavity in concrete, or a geological structure. However, the nonlinear problem is complicated, even if the nonlinear phenomena are calculated by the successive approximation method. The main difficulty is in the scattered field, which cannot be considered monochromatic. Even if the incident wave contains a single harmonic, higher ones will be generated in the layer. A more interesting problem is multifrequency incidence where one of the combination frequencies excites a resonant vibration of the layer. ${ }^{15}$ Finally, it is easy to reach a well-defined nonlinear response with a high-intensity pulse signal acting on the layer.

\section{GENERAL FORMULATION OF THE PROBLEM}

The statement of the problem is illustrated in Fig. 1. A plane layer with density $\rho_{0}$ and sound velocity $c_{0}$ is placed between $x=0$ and $x=h$. This layer is surrounded by another medium with density $\rho_{1}$ and sound velocity $c_{1}$. The relationship between the acoustical properties of both media can be arbitrary. Significant attention will be focused in the next sections on a soft and thin layer 


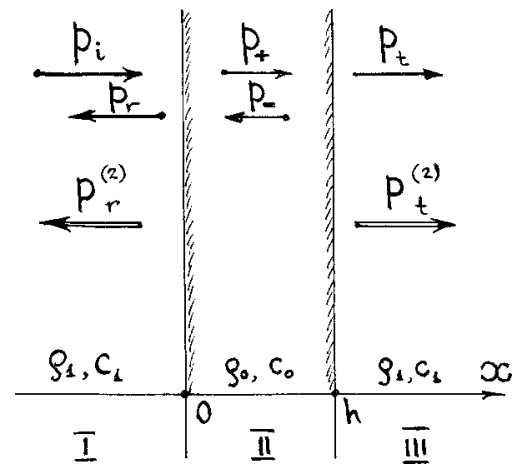

FIG. 1. Layer $(0<x<h)$, surrounding medium, and waves taken into account at the formulation of the problem.

$$
\zeta=\frac{c_{0} \rho_{0}}{c_{1} \rho_{1}} \ll 1, \quad \frac{\omega}{c_{0}} h \ll 1 .
$$

Also, the nonlinear properties of the layer will be taken into consideration.

It is presumed that the geometric nonlinearity (see, for example, Ref. 15, pp. 39-40) initiated from the structure of the equations of motion and continuity is small in comparison with the physical nonlinearity caused by the finite deformation of the layer. Therefore, these equations can be reduced to a linear connection between the variations of pressure $p$ and density $\rho$

$$
\nabla^{2} p-\frac{\partial^{2} \rho}{\partial t^{2}}=0
$$

But, the "equation of state"

$$
\rho=\rho(p),
$$

must be nonlinear. Equation (3) can be algebraic, for example a power series in $p$, or a more complicated function which is necessary in the case of strong nonlinearity. If the medium has a "memory," the Taylor series must be replaced by the corresponding functional expansion of VolterraFrechet type, ${ }^{15}$ pp. 80-81. Other models, for example accounting for the hysteretic properties, can be considered by a simplified approach in (2), (3).

The acoustic field in the linear medium surrounding the layer can be written as the sum of two waves traveling in opposite directions. In the region I, $-\infty<x<0$, the field consists of incident $p_{i}$ and reflected $p_{r}$ waves (see Fig. 1)

$$
\begin{aligned}
& p^{\mathrm{I}}=p_{i}\left(t-x / c_{1}\right)+p_{r}\left(t+x / c_{1}\right), \\
& \rho_{1} c_{1} u^{\mathrm{I}}=p_{i}\left(t-x / c_{1}\right)-p_{r}\left(t+x / c_{1}\right) .
\end{aligned}
$$

The field in the region III, $x>h$, is formed by the transmitted wave

$$
\begin{aligned}
& p^{\mathrm{III}}=p_{t}\left(t-(x-h) / c_{1}\right), \\
& \rho_{1} c_{1} u^{\mathrm{III}}=p_{t}\left(t-(x-h) / c_{1}\right) .
\end{aligned}
$$

When the nonlinear properties of the layer are significant, both the reflected $p_{r}$ and transmitted $p_{t}$ waves will contain nonlinear components, $p_{r}^{(2)}$ and $p_{t}^{(2)}$, respectively (see Fig. $1)$.
The acoustic field inside the layer (region II in Fig. 1) is described by the nonlinear equations (2) and (3). In the linear approximation it consists of right-going $p_{+}$and left-going $p_{-}$waves

$$
\begin{aligned}
& p^{\mathrm{II}}=p_{+}\left(t-x / c_{0}\right)+p_{-}\left(t+x / c_{0}\right), \\
& \rho_{0} c_{0} u^{\mathrm{II}}=p_{+}\left(t-x / c_{0}\right)-p_{-}\left(t+x / c_{0}\right) .
\end{aligned}
$$

The brief solution for the linear problem is given below to illustrate the mathematical technique and to derive the necessary formulas for the pulse response which will be useful for the nonlinear study.

Because most of this work deals with nonlinear pulse waves, we will avoid Fourier analysis also when solving the linear problem.

\section{LINEAR PULSE RESPONSE OF A LAYER}

To calculate the temporal profiles of both reflected and transmitted waves, the fields in the three regions (4)-(6) must be sewed together. The pressures and velocities must be equal at both boundaries, $x=0$ and $x=h$. These boundary conditions lead to the functional equations

$$
\begin{aligned}
& p_{i}(t)+p_{r}(t)=p_{+}(t)+p_{-}(t), \\
& \zeta\left[p_{i}(t)-p_{r}(t)\right]=p_{+}(t)-p_{-}(t), \\
& \rho_{+}\left(t-h / c_{0}\right)+p_{-}\left(t+h / c_{0}\right)=p_{t}(t), \\
& p_{+}\left(t-h / c_{0}\right)-p_{-}\left(t+h / c_{0}\right)=\zeta p_{t}(t),
\end{aligned}
$$

used in the calculation of the unknown profiles $p_{r}, p_{t}, p_{+}$, and $p_{-}$. The incident pulse $p_{i}(t)$ is assumed to be known.

The solution of the system (7)-(10) is easily found

$$
\begin{aligned}
& p_{+}(t)=\zeta(1+\zeta) \hat{G} \cdot \mathrm{e}^{\hat{D} h / c_{0}} \cdot p_{i}(t), \\
& p_{-}(t)=\zeta(1-\zeta) \hat{G} \cdot \mathrm{e}^{-\hat{D} h / c_{0}} \cdot p_{i}(t), \\
& p_{t}(t)=2 \zeta \hat{G} \cdot p_{i}(t), \\
& p_{r}(t)=-\left(1-\zeta^{2}\right) \hat{G} \cdot \sinh \left(\hat{D} h / c_{0}\right) \cdot p_{i}(t) .
\end{aligned}
$$

A symbolic operator-form representation is used, where

$$
\hat{G}=\left[\left(1+\zeta^{2}\right) \sinh \left(\frac{h}{c_{0}} \hat{D}\right)+2 \zeta \cos \left(\frac{h}{c_{0}} \hat{D}\right)\right]^{-1}, \quad \hat{D}=\frac{\partial}{\partial t},
$$

and $\mathrm{e}^{\tau \hat{D}}$ is a standard shift operator

$$
\mathrm{e}^{\tau \hat{D}} p(t) \doteq p(t+\tau) .
$$

The acoustic pressure field inside the layer (4) equals

$$
\begin{aligned}
p^{\mathrm{II}}(t)= & 2 \zeta\left\{\cosh \left[\left(\frac{h-x}{c_{0}}\right) \hat{D}\right]+\zeta \sinh \left[\left(\frac{h-x}{c_{0}}\right) \hat{D}\right]\right\} \cdot \hat{G} \\
& \cdot p_{i}(t) .
\end{aligned}
$$

For a single harmonic spectrum, $p_{i}=A_{i} \exp (-i \omega t)$, the operators rearrange into 


$$
\begin{aligned}
& \hat{D} \rightarrow-i \omega, \quad \cosh \left(\frac{h}{c_{0}} \hat{D}\right) \rightarrow \cos \left(\kappa_{0} h\right), \\
& \sinh \left(\frac{h}{c_{0}} \hat{D}\right) \rightarrow-i \sin \left(\kappa_{0} h\right),
\end{aligned}
$$

and the solution (17) takes the common form ${ }^{14}$

$$
p^{\mathrm{II}}=2 \zeta \frac{\zeta \sin \left[\kappa_{0}(h-x)\right]+i \cos \left[\kappa_{0}(h-x)\right]}{\left(1+\zeta^{2}\right) \sin \left(\kappa_{0} h\right)+i 2 \zeta \cos \left(\kappa_{0} h\right)} A_{i} e^{-i \omega t} .
$$

At significant difference in impedances, $\zeta \ll 1$ or $\zeta \gg 1$, the resonant properties of the layer are clearly defined at

$$
\kappa_{0} h=\pi n, \quad h=n \frac{\lambda}{2} \quad(n=1,2,3, \ldots) .
$$

The response of the layer is larger for the frequency components of the incident spectrum satisfying the condition (20).

A resonant behavior of a layer is favorable in the generation of new nonlinear frequencies. However, this problem calls for an extended and special investigation which is beyond the scope of the present work. On the other hand, NNDT often deals with nonresonant inclusions, which are small in comparison with the wavelength. To solve this important group of problems, a special limiting case of Eqs. (11) -(15) must be considered. Namely, both the ratio of impedances $\zeta(1)$ and wave thickness of layer $\omega h / c_{0}$ must simultaneously be small parameters. None is infinitesimally small, because the ratio $c_{0} \zeta / \omega h$ can be arbitrary in its magnitude. For such a thin inhomogeneity the solution (11)-(15) can be simplified with account for the inequalities (1)

$$
\begin{aligned}
& \hat{G}^{-1} \approx \frac{h}{c_{0}} \hat{D}+2 \zeta, \\
& \left(\frac{d}{d t}+\frac{2 c_{0} \zeta}{h}\right)\left(\begin{array}{c}
p_{ \pm} \\
p_{t} \\
p_{r}
\end{array}\right)=\left(\begin{array}{c}
c_{0} \zeta / h \\
2 c_{0} \zeta / h \\
-d / d t
\end{array}\right) p_{i}(t) .
\end{aligned}
$$

The most important properties of the response of a thin layer are the following ones.

The profile of the transmitted pulse equals

$$
p_{t}(t)=\frac{2 c_{0} \zeta}{h} \int_{-\infty}^{t} p_{i}\left(t^{\prime}\right) e^{-2 c_{0} \zeta\left(t-t^{\prime}\right) / h} d t^{\prime} .
$$

It follows from (23), that $h / 2 c_{0} \zeta$ plays the role of "relaxation time" of a layer. The combination $\omega h / 2 c_{0} \zeta=a^{-1}$ used later can be considered as a normalized relaxation time. The reflected pulse is calculated by the formula

$$
p_{r}(t)=-\frac{h}{2 c_{0} \zeta} \frac{d p_{t}}{d t}=p_{t}-p_{i} .
$$

One can see that the solutions (23) and (24) satisfy the energy conservation law for pulse response, namely

$$
\int_{-\infty}^{\infty} p_{i}^{2}(t) d t=\int_{-\infty}^{\infty}\left[p_{r}^{2}(t)+p_{t}^{2}(t)\right] d t .
$$

The fulfillment of the condition
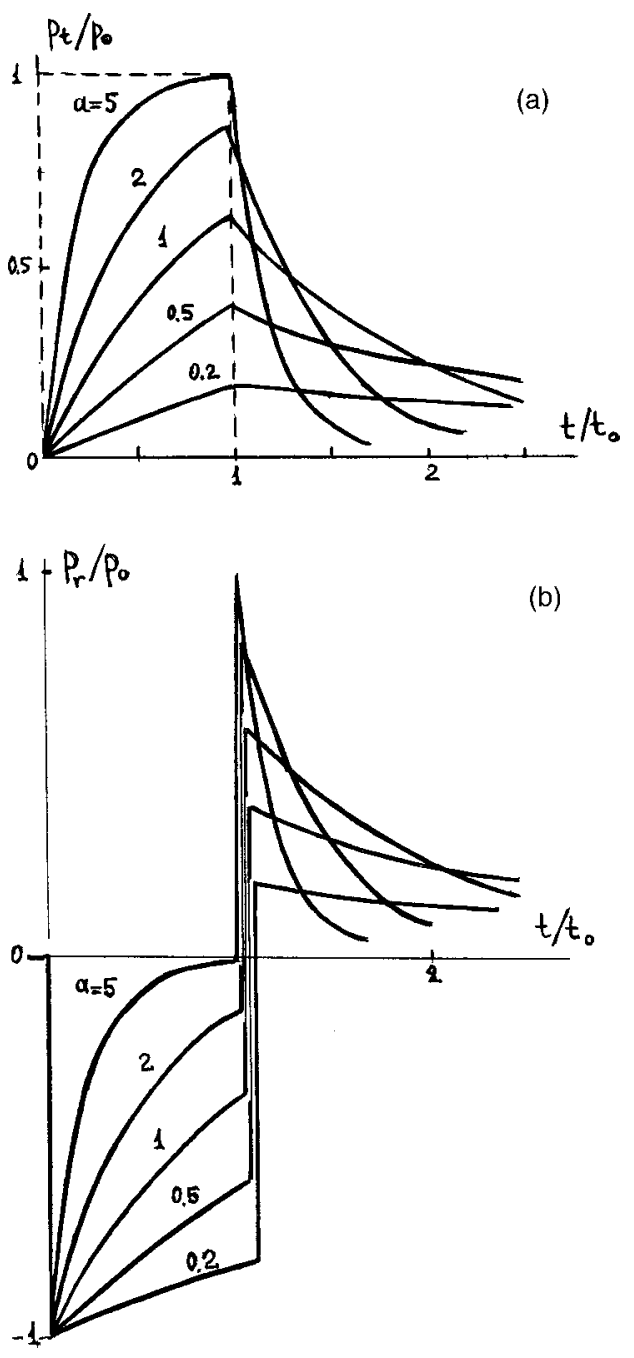

FIG. 2. Profiles of transmitted (a) and reflected (b) pulses at different values of the parameter $a=2 c_{0} t_{0} \zeta / h$. The incident pulse of rectangular form is shown by the dashed line.

$$
\int_{-\infty}^{\infty} p_{r}(t) p_{t}^{n}(t) d t=0
$$

means, in particular (at $n=1$ ), that the forms of reflected and transmitted pulses are orthogonal to each other.

The profiles of right- and left-going waves in the layer are equal, $p_{+}(t)=p_{-}(t)$. The inner acoustic field

$$
p^{\mathrm{II}}(t)=p_{t}(t)=p_{i}(t)+p_{r}(t),
$$

has the same time wave form as the transmitted pulse.

The profiles of the transmitted $p_{t}$ and reflected $p_{r}$ pulses are shown in Figs. 2(a) and (b) for different values of the parameter $a=2 c_{0} t_{0} \zeta / h$ equal to $0.2,0.5,1,2$, and 5 . Here, $t_{0}$ is the duration of the incident pulse of rectangular form shown by the dashed line in Fig. 2(a). It is significant that both the leading and the tail fronts of the monopolar transmitted pulse are smoothed out. On the other hand, the leading fronts of both negative and positive sections of the bipolar reflected pulse are as steep as the front of the incident pulse.

It is convenient to keep in mind some numerical estimations. If a gaseous layer (air) is surrounded by a denser medium (water), $\zeta \approx 3 \cdot 10^{-4}$, the parameter $a=1$ corresponds to the ratio $h / t_{0}=20 \mathrm{~cm} / \mathrm{s}$. For example, the width of a layer 

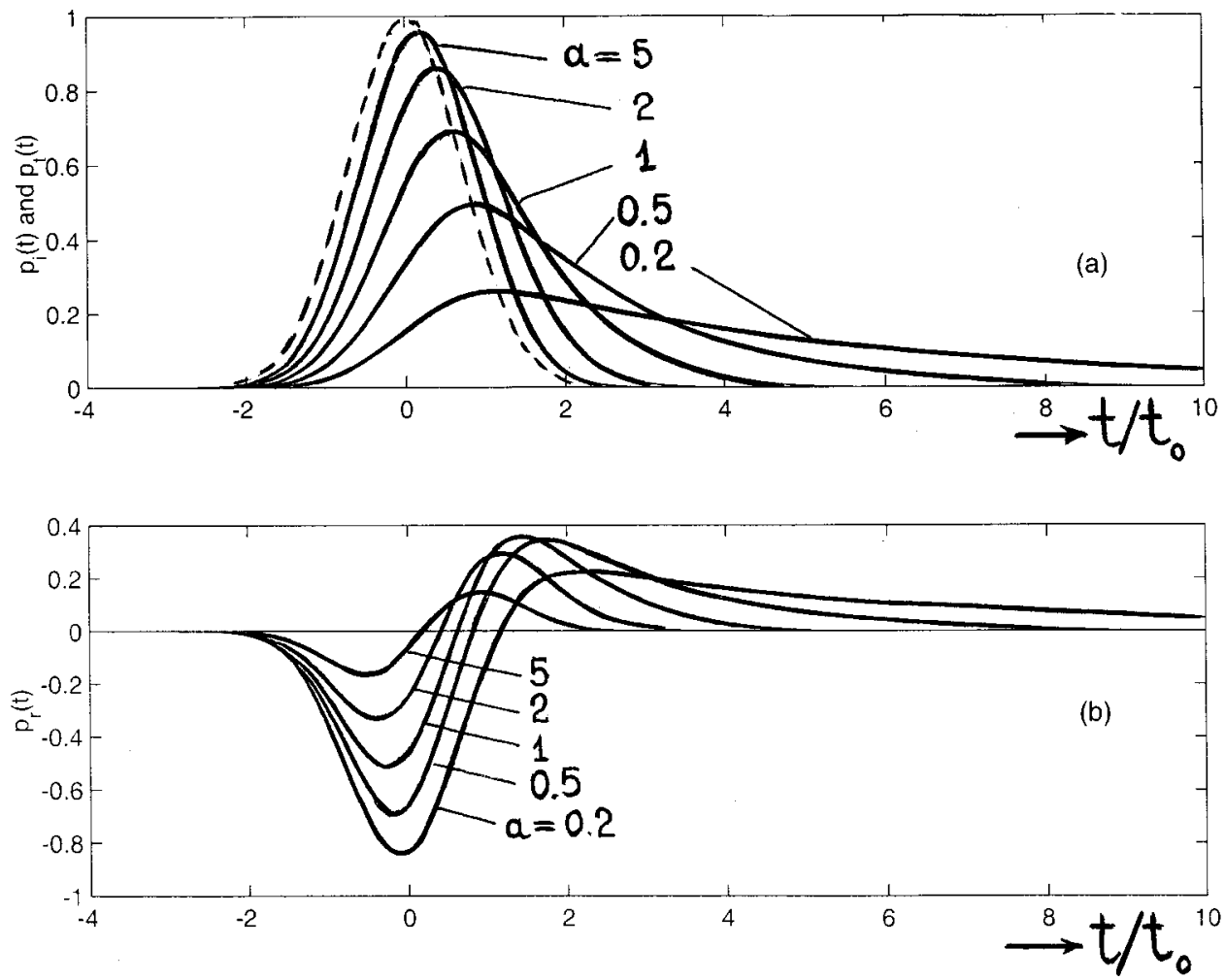

FIG. 3. Response to Gaussian pulse (dashed line): forms of transmitted (a) and reflected (b) pulses. $h \sim 0.01 \mathrm{~cm}$ corresponds to a duration of the probing pulse in the order of $0.5 \mathrm{~ms}$.

In Fig. 3 the reflected and transmitted pulses are shown for the same parameters $a$, as in Fig. 2, but for a Gaussian incident wave

$$
p_{i}=p_{0} \exp \left(-t^{2} / t_{0}^{2}\right) ;
$$

see the dashed line in Fig. 3(a). The main peculiarities of the pulse response exhibited in Fig. 2 can also be seen in Fig. 3.

It should be emphasized that the simple analytical results (22)-(24) shown in Figs. 2 and 3 are derived with account for both inequalities (1). The small parameters (1) are presented in all solutions in the form of a ratio, equal to $a$ $=2 c_{0} t_{0} \zeta / h$ for pulse signals, and $a=2 c_{0} \zeta / h \omega$ for harmonic signals. This ratio of two small numbers can be arbitrary in magnitude.

If the ratio of impedances $\zeta(1)$ is very small and $a$ $\rightarrow 0$, it follows from the solution (23), (24) that $p_{t}(t) \rightarrow 0$, and $p_{r}(t) \rightarrow-p_{i}(t)$; this limiting case corresponds to reflection from a free boundary. In the opposite situation, if the thickness $h$ of the layer is very small and $a \rightarrow \infty$, it follows from (23), (24) that $p_{t}(t) \rightarrow p_{i}(t)$, and $p_{r}(t) \rightarrow 0$; this second limit corresponds to complete transmission of the incident wave through the layer.

When assuming a temporal dependence of $p_{i}, p_{r}$, and $p_{t}$ of the form $\exp (-i \omega t)$, the transmission and reflection coefficients are calculated to be

$$
K_{t}(\omega)=i \frac{2 c_{0} \zeta / \omega h}{1+i 2 c_{0} \zeta / \omega h}, \quad K_{r}(\omega)=-\frac{1}{1+i 2 c_{0} \zeta / \omega h} .
$$

One can see that the high frequencies in the pulse spectrum are reflected quite well, because $K_{r} \rightarrow-1$ at $\omega \rightarrow \infty$. So, the steep leading front of the rectangular incident pulse reflects completely from the layer, as is shown in Fig. 2(b).

The last linear example is devoted to the response of a layer to a sinusoidal incident pulse of finite duration, $0<t$ $<t_{0}$. The form of the transmitted wave is

$$
\begin{aligned}
\frac{p_{t}}{p_{0}}= & \frac{a^{2}}{1+a^{2}} \sin \omega t+\frac{a}{1+a^{2}}\left(e^{-a \omega t}-\cos \omega t\right), \quad 0<t<t_{0}, \\
\frac{p_{t}}{p_{0}}= & \frac{a^{2}}{1+a^{2}} e^{-a \omega\left(t-t_{0}\right)} \cdot \sin \omega t_{0}+\frac{a}{1+a^{2}} e^{-a \omega t} \\
& \times\left(1-e^{a \omega t_{0}} \cos \omega t_{0}\right), \quad t>t_{0} .
\end{aligned}
$$

As distinct from monopolar incident pulses, the notation for $a$ is here $a=2 c_{0} \zeta / \omega h$. When $t_{0} \rightarrow \infty$ and $a \omega t \gg 1$, the result is a steady-state harmonic response

$$
\begin{aligned}
\left(\begin{array}{c}
p_{t} \\
p_{r}
\end{array}\right)= & -\frac{p_{0}}{\sqrt{\omega^{2}+\left(2 c_{0} \zeta / h\right)^{2}}}\left(\begin{array}{c}
2 c_{0} \zeta / h \\
\omega
\end{array}\right)\left(\begin{array}{c}
\cos \\
\sin
\end{array}\right) \\
& \times\left(\omega t+\arctan \frac{2 c_{0} \zeta}{\omega h}\right) .
\end{aligned}
$$

The amplitude of the transmitted wave decreases with increase in frequency $\omega$ (from $p_{0}$ to 0 ). The amplitude of the reflected wave increases monotonically and tends to $p_{0}$ when $\omega \rightarrow \infty$.

\section{WEAKLY NONLINEAR THIN LAYER}

If the width of the layer $h$ is small in comparison with the wavelength, the inner pressure will be equal to the outer one; in other words: the connection (27) is true also for the nonlinear deformation of the layer 


$$
p^{\mathrm{I}}=p_{i}(t)+p_{r}(t)=p^{\mathrm{II}}=p^{\mathrm{III}}=p_{t}(t) .
$$

The boundary conditions (8) and (10) for the particle velocity will be expressed as

$\frac{1}{\rho_{1} c_{1}}\left[p_{i}(t)-p_{r}(t)\right]=u(x=0, t), \quad \frac{1}{\rho_{1} c_{1}} p_{t}(t)=u(x=h, t)$.

It follows from (32) and (33) that the difference of velocities equals

$$
u(h, t)-u(0, t)=\frac{2}{\rho_{1} c_{1}}\left(p^{\mathrm{II}}-p_{i}\right) .
$$

On the other hand, the nonlinear dependence $u(p)$ can be written by means of the linear equation of discontinuity, used at the derivation of (2), and the nonlinear "equation of state" (3)

$$
\operatorname{div} \mathbf{u}=-\frac{1}{\rho_{0}} \frac{\partial}{\partial t} \rho(p) .
$$

For a thin layer (35) takes the form

$$
u(h, t)-u(0, t) \approx-\frac{h}{\rho_{0}} \frac{d}{d t} \rho\left(p^{\mathrm{II}}\right) .
$$

We hereafter symbolize the inner field $p^{\mathrm{II}} \equiv p$. As (34) is equal to (36) we derive the nonlinear equation for the inner field

$$
\frac{d}{d t} f(p)+\frac{2 c_{0} \zeta}{h} p=\frac{2 c_{0} \zeta}{h} p_{i}(t), \quad \rho\left(p^{\mathrm{II}}\right) \equiv \frac{1}{c_{0}^{2}} f(p) .
$$

The new equation (37) is a generalization of (22) when taking into account the nonlinear properties of the layer. If the inner field $p(t)$ is known, the reflected and transmitted pulses are known as well

$$
p_{r}(t)=p(t)-p_{i}(t), \quad p_{t}(t)=p(t) .
$$

For weak nonlinearity, the function $f$ can be modeled by a series expansion

$$
f(p) \approx p-\frac{\epsilon}{c_{0}^{2} \rho_{0}} p^{2}+\frac{\chi}{\left(c_{0}^{2} \rho_{0}\right)^{2}} p^{3}+\cdots,
$$

where $\epsilon$ and $\chi$ are the coefficients of quadratic and cubic nonlinearities, respectively. In this case, (37) can be solved by the successive approximation method: $p=p^{(1)}+p^{(2)}$ $+\cdots$. Taking into account only the quadratic term in (39), we obtain a pair of equations for $p^{(1)}$ and $p^{(2)}$

$$
\begin{aligned}
& \frac{d p^{(1)}}{d t}+\frac{2 c_{0} \zeta}{h} p^{(1)}=\frac{2 c_{0} \zeta}{h} p_{i}, \\
& \frac{d p^{(2)}}{d t}+\frac{2 c_{0} \zeta}{h} p^{(2)}=\frac{\epsilon}{c_{0}^{2} \rho_{0}} \frac{d}{d t} p^{(1)^{2}} .
\end{aligned}
$$

Equation (40) coincides in the first approximation with (22) for the linear transmitted wave $p_{t}$. The second-order response is described by an analogous equation, but the "driving force" in the right-hand side of (41) is determined by the 1 st approximation solution $p^{(1)}$, instead of the incident pulse $p_{i}$ in Eq. (40).

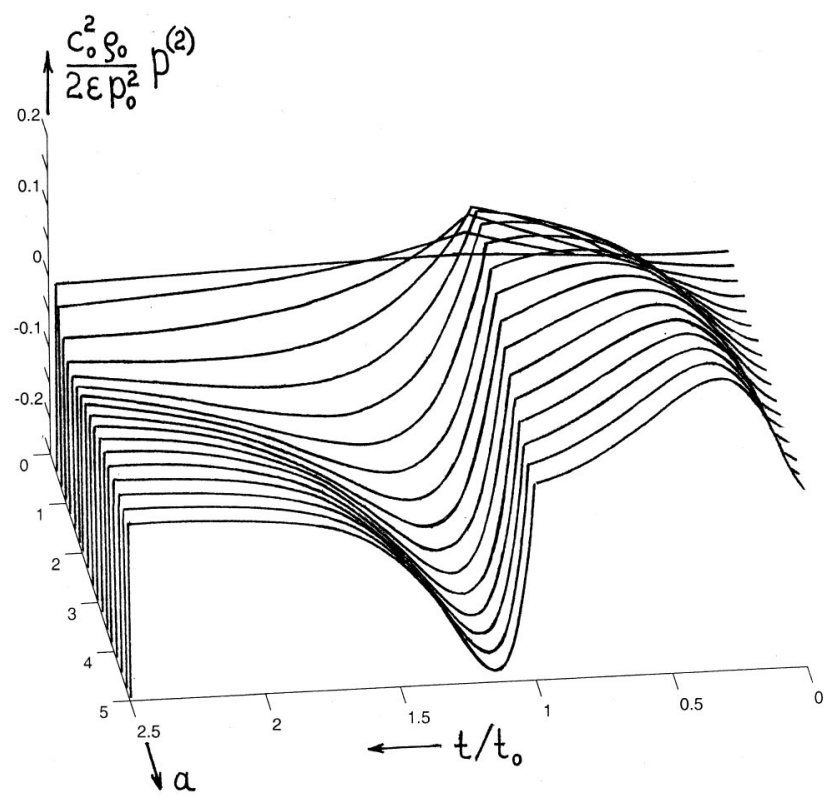

FIG. 4. Weak nonlinear response to rectangular incident pulse. The linear part of response is shown in Fig. 2.

It follows from (41) that the nonlinear pulse is described by the function $p^{(2)}(t)$ whose integral area equals zero. If the monopolar incident pulse has rectangular form, one can calculate the nonlinear response using the solution for $p_{t}(t)$ [see Fig. 2(a)] as the first approximation

$$
\begin{aligned}
p^{(2)}= & \frac{2 \epsilon p_{0}^{2}}{c_{0}^{2} \rho_{0}} e^{-a \tau} \\
& \times\left\{\begin{array}{l}
\left(e^{-a \tau}-1+a \tau\right), \quad 0<\tau<1, \\
\left(e^{-a}-1+a\right)-\left(e^{a}-1\right)^{2}\left(e^{-a}-e^{-a \tau}\right), \quad \tau>1 .
\end{array}\right.
\end{aligned}
$$

Here, the parameter $\tau=t / t_{0}$ and $a=2 c_{0} t_{0} \zeta / h$. The profiles of the bipolar nonlinear pulse (42) are shown in Fig. 4 for different values of $a$.

Using the solution (30) as the first approximation, we derive the steady-state nonlinear response to a sinusoidal pulse

$$
\begin{aligned}
p^{(2)}= & \frac{\epsilon p_{0}^{2}}{c_{0}^{2} \rho_{0}} \frac{\omega h / 2 c_{0} \zeta}{1+\left(\omega h / 2 c_{0} \zeta\right)^{2}} \frac{1}{\sqrt{1+\left(\omega h / c_{0} \zeta\right)^{2}}} \\
& \cdot \cos \left[2 \omega t+2 \arctan \left(\frac{2 c_{0} \zeta}{\omega h}\right)+\arctan \left(\frac{c_{0} \zeta}{\omega h}\right)\right] .
\end{aligned}
$$

It is important at this point to evaluate the amplitude of the nonlinear response (43). It reaches its maximum value at

$$
\frac{\omega h}{2 c_{0} \zeta} \approx 0.45, \quad p_{\max }^{(2)} \approx 0.3 \frac{\epsilon p_{0}^{2}}{c_{0}^{2} \rho_{0}} .
$$

This is a nonresonant and smooth maximum caused by competition between nonlinear growth of $p^{(2)}$ and the inner field which decays more rapidly with increase in frequency. In the absence of an air layer, a corresponding thin layer of another homogeneous medium can generate a weak second harmonic with the amplitude ${ }^{15}$ 


$$
p_{h}=\frac{\epsilon_{1}}{2 c_{1}^{3} \rho_{1}} \omega p_{0}^{2} h .
$$

This formula can be easily derived from the Bessel-Fubini solution (see, for example, Refs. 1 and 15, p. 31), or from Riemann solution at distances $h$ small in comparison with shock formation distance. The ratio of amplitudes (44) and (45) can reach its maximum possible value

$$
\frac{p_{\max }^{(2)}}{p_{h}} \approx \frac{2}{3} \frac{\epsilon}{\epsilon_{1}}\left(\frac{c_{1}^{2} \rho_{1}}{c_{0}^{2} \rho_{0}}\right)^{2},
$$

at $\kappa_{0} h \approx 0.9 \zeta$.

For an air layer, surrounded by water, the ratio (46) is evaluated as $10^{7}$. The huge increase in the nonlinear properties of a medium containing highly compressible inclusions is impressive, but caution must be exercised in the interpretation of such estimates. For example, the ratio of maximum nonlinear response (44) to an incident amplitude $p_{0}$ can be in the order of 1 at pressure $p_{0} \sim 20 \mathrm{~atm}$. Such pressure corresponds in water to a sound intensity of about $10^{2} \mathrm{~W} / \mathrm{cm}^{2}$, which is difficult to reach in normal high-power ultrasound experiments. However, there is no problem to form an acoustic pulse with a peak pressure on the order of several hundreds of atmospheres. ${ }^{16}$

A plane cloud of floating bubbles is often used in experiments to create a highly nonlinear region in water. For this purpose, electrolytic decomposition of water molecules into hydrogen and oxygen can be utilized, as well as pumping of compressed air through orifices in a pipeline lying on the bottom. ${ }^{1-3}$ Let the width $h$ of the layer be less than the wavelength, and the volume concentration $v$ of air be small. Then, the density of the bubbly liquid is about the density of pure water. It follows from (34) and (36) that

$$
\frac{d}{d t} \rho(p)+\frac{2}{c_{1} h} p=\frac{2}{c_{1} h} p_{i}(t),
$$

where the variation of density caused by the incident pulse $p_{i}$ is connected only with the compressibility of air

$$
\rho=-\rho_{1} n V .
$$

Here, $n$ is the number of bubbles per unit volume, and $V$ is the variation of the volume of a single bubble described by the equation (see, for example, Ref. 15, p. 160)

$$
\frac{d^{2} V}{d t^{2}}+\omega_{0}^{2}\left(V-g V^{2}\right)=-\nu p .
$$

The following notations are used here:

$$
\omega_{0}^{2}=\frac{3 c_{0}^{2}}{R_{0}^{2}}, \quad g=\frac{3 \epsilon}{4 \pi R_{0}^{3}}, \quad \nu=\frac{4 \pi R_{0}^{2}}{\rho_{0}},
$$

where $R_{0}$ is the equilibrium radius of the air bubble. Combining Eqs. (47)-(49), one can derive the equation governing the response of a bubble inside the layer for an incident pulse

$$
\frac{d^{2} V}{d t^{2}}+\frac{1}{2} \nu \rho_{1} c_{1} n h \cdot \frac{d V}{d t}+\omega_{0}^{2}\left(V-g V^{2}\right)=-\nu p_{i} .
$$

The second term on the left-hand side of (51) is connected with energy lost in the excitation of reflected and transmitted waves. The $Q$-factor for the oscillatory system (51)

$$
Q=\frac{2 \omega_{0}}{\nu \rho_{1} c_{1} n h}=\frac{2}{\sqrt{3}} \frac{c_{0} \rho_{0}}{c_{1} \rho_{1}} \frac{R_{0}}{h v}, \quad v=n \cdot \frac{4}{3} \pi R_{0}^{3},
$$

is small in many instances, and then the second derivative can be eliminated. In this case the system (47)-(49) can be reduced to the equation

$$
\frac{d}{d t}\left(p-\frac{\epsilon}{c_{0}^{2} \rho_{0}} p^{2}\right)+\frac{2 c_{0} \zeta}{h v} p=\frac{2 c_{0} \zeta}{h v} p_{i}(t),
$$

which differs in notations only from (37), accounting only for quadratic nonlinearity. The ratio of nonlinear responses (44) and (45) is described by an equation similar to (46), but its right-hand side must be multiplied by the volume concentration $v$ of the gas. Consequently, the evaluation (46) for $v=10^{-4}$ gives an increase in nonlinearity of $10^{3}$ times (instead of $10^{7}$ indicated above). This value corresponds to measurements. ${ }^{1}$

Considering the more exotic case when $Q>1$, which means that a cloud of bubbles can execute free quasiperiodic oscillations, the power expansion $\rho(p)$ (39) must be replaced by a functional series of Volterra-Frechet type

$$
\begin{aligned}
\rho(p)= & \frac{\rho_{1} n \nu}{\omega_{0}} \int_{0}^{\infty} \sin \left(\omega_{0} t_{1}\right) p\left(t-t_{1}\right) d t_{1}-\frac{\rho_{1} n g \nu^{2}}{\sqrt{\omega_{0}^{2}-\delta^{2}}} \\
& \times \int_{0}^{\infty} \int_{0}^{\infty} \int_{0}^{\infty} \mathrm{e}^{-\delta t_{1}} \sin \left(\sqrt{\omega_{0}^{2}-\delta^{2}} t_{1}\right) \sin \left(\omega_{0} t_{2}\right) \\
& \times \sin \left(\omega_{0} t_{3}\right) \cdot p\left(t-t_{1}-t_{2}\right) \cdot p\left(t-t_{1}-t_{3}\right) d t_{1} d t_{2} d t_{3},
\end{aligned}
$$

which can be derived from (47)-(49). For example, if the incident pressure varies according to $p_{i}=p_{0} \sin (\omega t)$, the nonlinear response, in accordance with the equation of state (54), will be a vibration at the frequency $2 \omega$

$$
p^{(2)}=\frac{\left(g \omega_{0}^{2} \nu p_{0}^{2}\right) \cdot(2 \delta \omega) \cdot \cos \left(2 \omega t+2 \phi_{1}+\phi_{2}\right)}{\left[\left(\omega_{0}^{2}-\omega^{2}\right)^{2}+(2 \delta \omega)^{2}\right] \sqrt{\left(\omega_{0}^{2}-4 \omega^{2}\right)^{2}+(4 \delta \omega)^{2}}},
$$

where

$\delta=\frac{1}{2} \nu \rho_{1} c_{1} n h, \quad \tan \phi_{1}=\frac{\omega^{2}-\omega_{0}^{2}}{2 \delta \omega}, \quad \tan \phi_{2}=\frac{\omega_{0}^{2}-4 \omega^{2}}{4 \delta \omega}$.

The second example of nonlinear layer illustrating the general approach is the contact between two rough surfaces of solids. ${ }^{17,18}$ It can be modeled by a thin layer whose linear and nonlinear elastic moduli are determined by a probabilistic distribution of micro-asperity height of $w(l)^{18}$

$$
p=\kappa\left(h_{0}-h\right)+\frac{\beta}{2}\left(h_{0}-h\right)^{2},
$$

where

$$
\kappa=E \int_{h_{0}}^{l_{0}} w(l) \frac{d l}{l}, \quad \beta=E \frac{w\left(h_{0}\right)}{h_{0}},
$$

where $E$ is Young's modulus of the solid, $l_{0}$ is the maximum height of micro-asperity, $h_{0}(P)$ is the equilibrium thickness 
of contact (in the absence of acoustic waves), which depends on the static pressure $P$ applied to the contact. The reflection and transmission factors are dependent on $P$, and the nonlinear factors have extreme magnitudes at the specific pressure $P=P_{*}$. Using a measured dependence of the reflected wave on $P$, one can reconstruct the probability distribution $w(l)$ and evaluate the surface roughness. ${ }^{18}$

For the rough contact (57) and (58), Eq. (37) takes the form

$$
\frac{d}{d t}\left(p-\frac{\beta}{2 \kappa^{2}} p^{2}\right)-\frac{2 \kappa}{\rho_{1} c_{1}} p=\frac{2 \kappa}{\rho_{1} c_{1}} p_{i} .
$$

An earlier discussed technique for evaluation of surface quality ${ }^{18}$ was based on the steady-state response at frequencies $\omega$ and $2 \omega$. Equation (59) offers the possibility to calculate the nonlinear pulse response as well.

\section{STRONGLY NONLINEAR LAYER}

Equation (37) can be solved exactly for several types of nonlinear dependence $f(p)$. Some of these solutions can be related to real inhomogeneities. An important example is

$$
\frac{p}{p_{*}}=\exp (b f)-1,
$$

which demonstrates the nonlinear connection between pressure and density (equivalent to a nonlinear stress-strain relationship) typical for strongly loaded solids. At increase in $p$ the function $f$ (proportional to the variation of density of the medium) increases slower than one following a linear law. Such behavior demonstrates a rise in rigidity, showing the nonlinear character of compressibility. Alternatively, an increase in the stretching load leads to fast variation of density: $f \rightarrow-\infty$ at $p$ tending to the finite rarefaction $p=-p_{*}$. The nonlinear dependence (60) can be expanded in power series for weak deviations of pressure and density from the equilibrium state

$$
\frac{p}{p_{*}}=b f+\frac{1}{2} b^{2} f^{2}+\frac{1}{6} b^{3} f^{3}+\cdots .
$$

A comparison between the expansions (61) and (39) demonstrates the meaning of the weak quasilinear deformation constants

$$
b p_{*}=1, \quad b=\frac{2 \epsilon}{c_{0}^{2} p_{0}}, \quad b^{2}=\frac{3 \chi}{\left(c_{0}^{2} p_{0}\right)^{2}}, \cdots .
$$

For a periodic signal $p_{i}(t)=p_{0} \sin (\omega t)$, the exact steadystate solution of (37) with account for (60) equals

$$
\frac{p}{p_{*}}=\frac{\exp [-a \beta \cos (\omega t)]-S}{\exp [-a \beta \cos (\omega t)]+S}
$$

where

$$
S=I_{0}(a \beta)+2 \sum_{n=1}^{\infty}(-1)^{n} I_{n}(a \beta) \cos \left(n \omega t-2 \arctan \frac{n}{a}\right) .
$$

Here, $I_{n}$ are modified Bessel functions

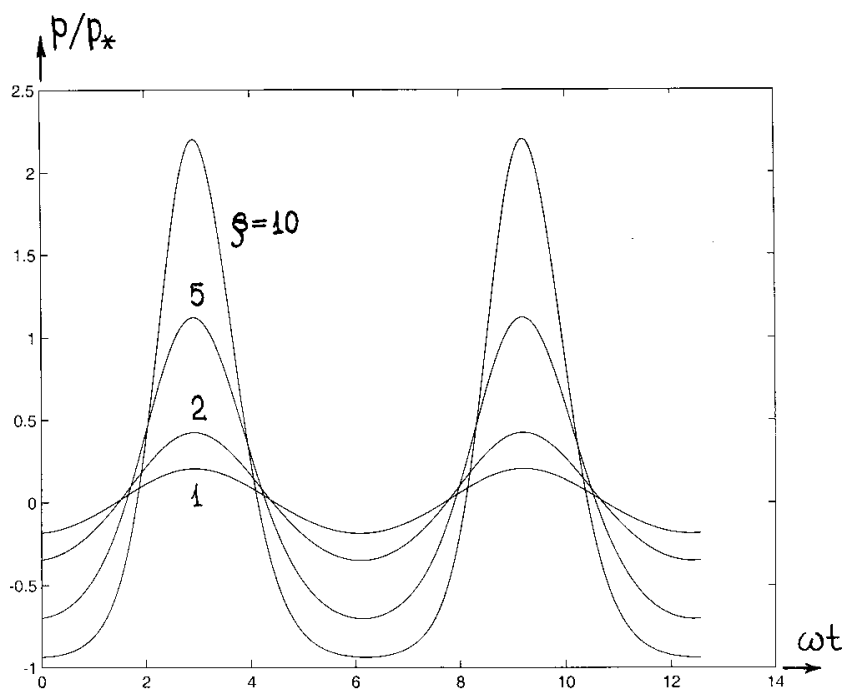

(a)

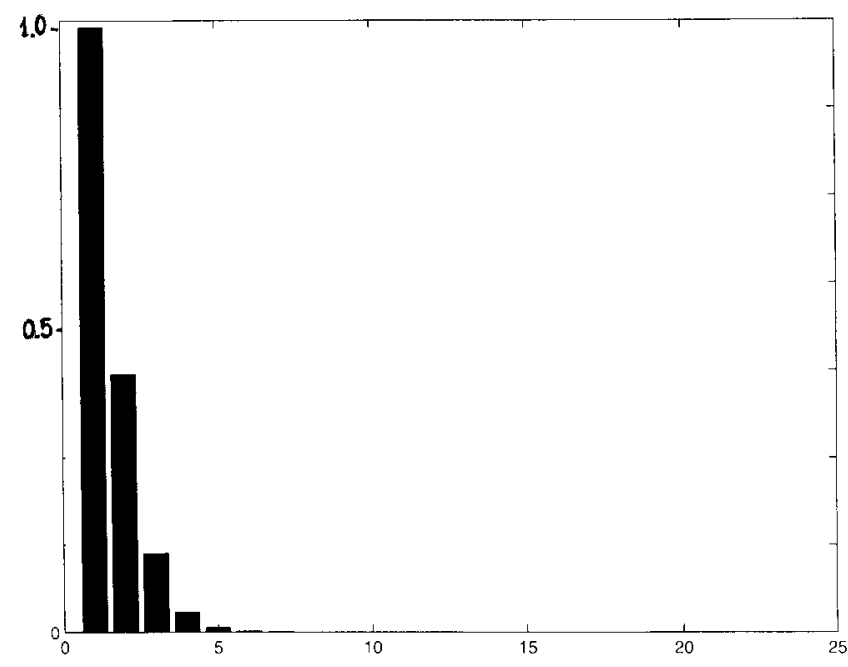

(b)

FIG. 5. Strong nonlinear response to harmonic incident signal. Temporal profiles are shown for $a=0.2, b p_{*}=1$ and different values of $\beta$ (59), (64) (a). The spectrum corresponding to the curve $\beta=10$ is given in (b).

$$
a=\frac{2 c_{0} \zeta}{\omega h} b p_{*}, \quad \beta=\frac{p_{0}}{p_{*}} .
$$

The parameter $\beta$ is a ratio of the amplitude $p_{0}$ of the incident wave to the internal pressure $p_{*}$ of medium characterizing the nonlinear properties of the stress-strain relationship (60). As the parameter $\beta$ increases, the nonlinear effects must increase as well. Distortion of profile and higher harmonics generation will be more pronounced for rarefaction half-periods, because tensile loads cause fractures at lower magnitudes than compressive loads.

The temporal profiles of strong acoustic response and their corresponding frequency spectra are shown in Fig. 5 and Fig. 6. The numerical values of parameters were $b p_{*}$ $=1, a=0.2$ in Fig. 5, and $a=5$ in Fig. 6 . The profiles are presented in Fig. 5(a) and Fig. 6(a) for different $\beta$ equal to 1, 2,5 , and 10. One can see that increase in both $a$ and $\beta$ leads to increase in the nonlinear distortion. For $\beta=1$ the profiles in both Fig. 5(a) and Fig. 6(a) are quasiharmonic. With increase in $\beta$ their amplitude increases, and at $\beta=10$ the shape 

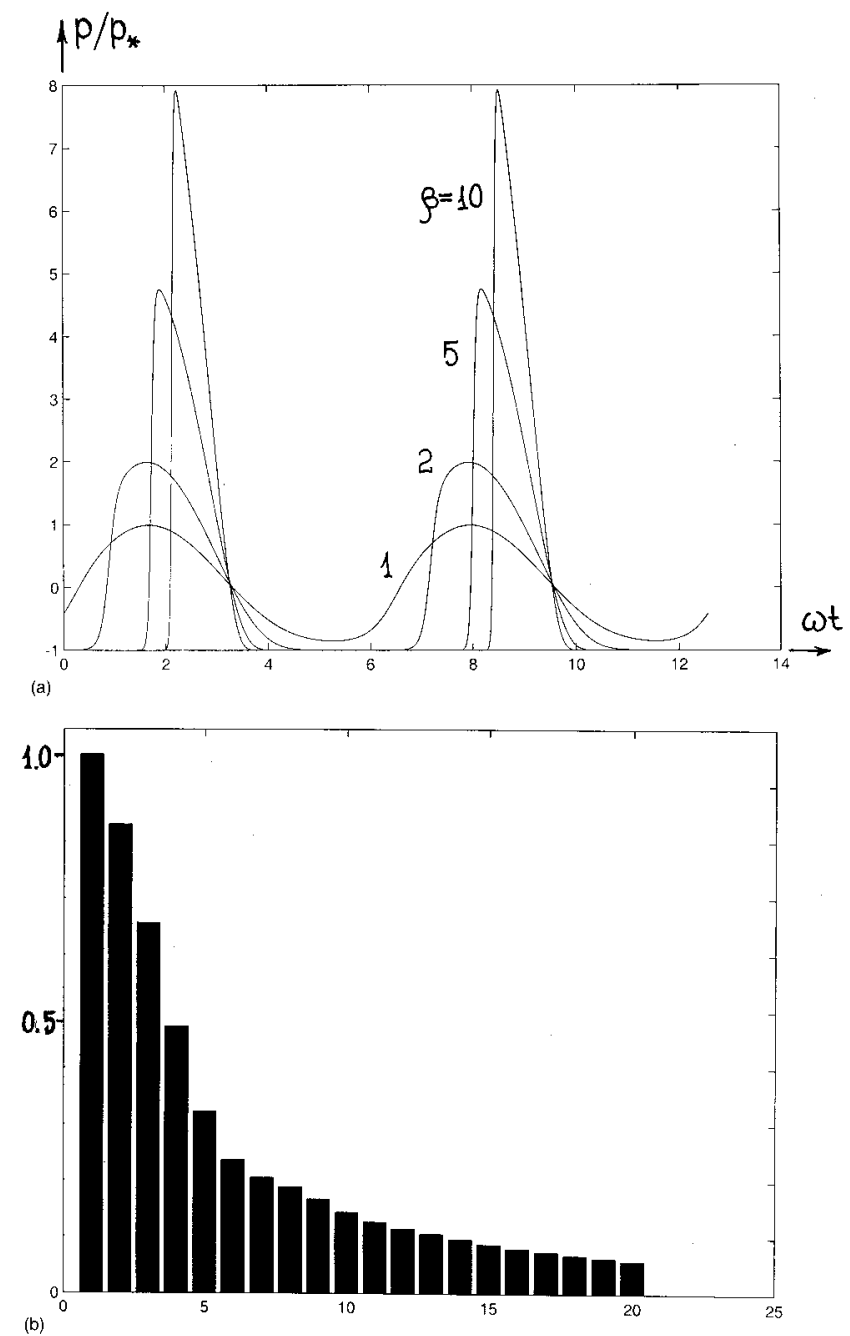

FIG. 6. The same as in Fig. 5, but for $a=5$.

becomes unrecognizable. The sections of positive and negative pressure are distorted quite differently. The section of positive pressure has a structure of a high and sharp peak, and the leading front of it is steep. On the other hand, the section of negative pressure is smoothed. In accordance with the relationship $p(\rho)$ in $(60)$ the normalized negative pressure $p / p_{*}$ cannot be less than -1 .

The spectra in Fig. 5(b) and Fig. 6(b) are calculated for profiles corresponding to the highest nonlinearity, $\beta=10$. In the example with $a=0.2$ [Fig. 5(b)] only 5 harmonics are discernible, but when $a=5$ [Fig. 6(b)] the number of harmonics reaches several tens, and the amplitudes of the second and third ones are comparable in magnitude with the fundamental.

A similar nonlinear behavior of profiles and spectra was observed experimentally ${ }^{17}$ for a layer formed by contact of two rough solid boundaries.

Also, the non-steady-state response can be calculated analytically. Let the incident acoustic pressure vary as $p_{i}$ $=p_{0} \cdot \phi(t)$, where $\phi$ is the function describing the shape of the incident pulse. The solution of the problem (37), (60) can be written as

$$
\frac{p}{p_{*}}=\frac{\exp \left[a \tau+a \beta \int_{-\infty}^{t} \phi(x) d x\right]}{C+\int_{-\infty}^{\tau} \exp \left[a x+a \beta \int_{-\infty}^{x} \phi\left(x_{1}\right) d x_{1}\right] d x}-1,
$$

where

$$
\tau=\frac{t}{t_{0}}, \quad a=\frac{2 c_{0} t_{0} \zeta}{h} b p_{*} .
$$

Here, $t_{0}$ is a characteristic duration of pulse, and $C$ is an arbitrary constant which must be determined on the basis of the causality principle. Namely, the leading front of response cannot appear earlier than the front of the incident pulse. The solution (66) can be reduced to a simple integral form if the incident pulse has a shape given by

$$
\phi= \pm \frac{\tau}{1+\tau^{2}} \Xi(\tau)
$$

Here, $\Xi$ is the Heaviside step function indicating that the disturbance $\phi$ is identically zero at $\tau<0$. The sign "plus" corresponds to a monopolar pulse of positive pressure, and "minus" to a negative one. The solution (66), (68) takes the form

$$
\frac{p}{p_{*}}=\frac{\left(1+\tau^{2}\right)^{ \pm(1 / 2) a \beta} e^{a \tau}}{1+a \int_{0}^{\tau} e^{a x}\left(1+x^{2}\right)^{ \pm(1 / 2) a \beta} d x}-1 .
$$

The nonlinear pulse response (69) is shown in Fig. 7 and Fig. 8 by solid lines. The corresponding linear pulses are shown by dashed curves for the comparison; their shape was calculated from the formula (23). For all pulses in Fig. 7 the parameter $a$ equals 0.2 , and for the ones in Fig. $8 a=5$.

The other parameter $\beta=p / p_{*}$ was put to $1,2.5$, and 5 for the three sets of curves at each figure.

One should note the difference in the distortion between positive (a) and negative (b) pulses. An incident rarefaction pulse is deformed more strongly than a compression pulse, and the influence of nonlinear properties on its shape is stronger.

When the incident pulse is very short, in the form of a $\delta$ function, Eq. (37) becomes

$$
\frac{d}{d \tau} f(p)+a p=a p_{0} \delta(\tau), \quad a=\frac{2 c_{0} t_{0} \zeta}{h}, \quad \tau=t / t_{0} .
$$

This can be solved for an arbitrary stress-strain relationship $f(p)$. One can solve the homogeneous equation (70), because its right-hand side equals zero for any moment of time, except at $\tau=0$

$$
F(p) \equiv \int \frac{d f(p)}{p}=-a \tau+C, \quad p=F^{-1}(-a \tau+C) .
$$

Following from (70), the constant $C$ must be determined from the condition

$$
\int_{-\infty}^{\infty} F^{-1}(-a \tau+C) d \tau=p_{0}
$$

In particular, for the nonlinear dependence $f=p_{*} \ln (1$ $+p / p_{*}$ ) [discussed above, see (60)], the general formula (71) reduces to the simple result

$$
\frac{p}{p_{*}}=\frac{\left(1-e^{-a p_{0} / p_{*}}\right) e^{-a \tau}}{1-\left(1-e^{-a p_{0} / p_{*}}\right) e^{-a \tau}},
$$




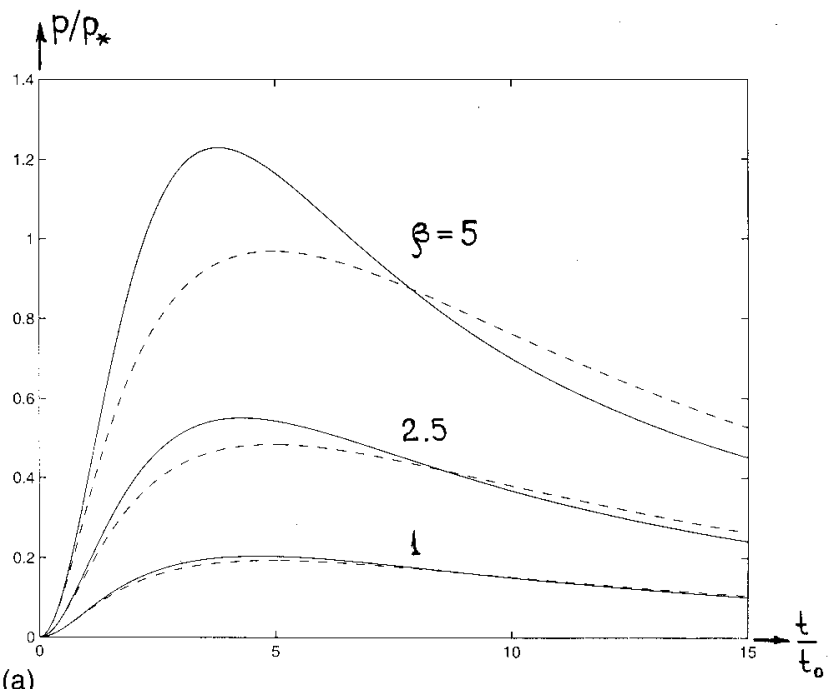

(a)

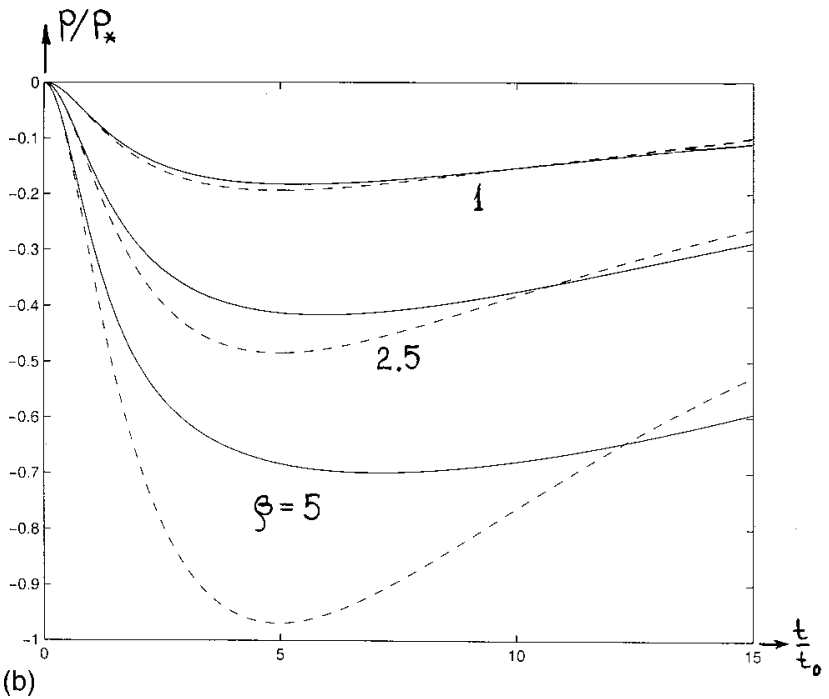

FIG. 7. Strong nonlinear response to a single monopolar incident pulse of compression (a) and rarefaction (b) for $a=0.2$ and different values of $\beta$. The corresponding linear response is shown by dashed lines.

which is valid for $p>0$ and $p_{0}<0$. The response (73) reaches its extremum

$$
\left(\frac{p}{p_{*}}\right)_{\mathrm{ext}}=-1+\exp \left(a \frac{p_{0}}{p_{*}}\right),
$$

at the instant $\tau=0$ when the incident $\delta$ pulse acts on the layer. With increase in time $\tau$ the absolute value of the response (73) decreases monotonically.

The temporal profiles of the pulse (73) are shown in Fig. 9 for negative $p_{0}$; if $-a p_{0} / p_{*}$ is small, $p(\tau)$ is near to the exponent. With increase in $-a p_{0} / p_{*}$, the response starts with the value $p / p_{*} \approx-1$. After a perceptible delay it tends to zero.

Despite that this work is devoted to the direct nonlinear problem, it is pertinent to make some remarks on the possibility of using these results in the solution of inverse nonlinear problems. Many spectral or temporal peculiarities of the nonlinear response can serve as distinguishing characteristics useful for nonlinear nondestructive testing (NNDT). Only the simplest possibilities are discussed here.
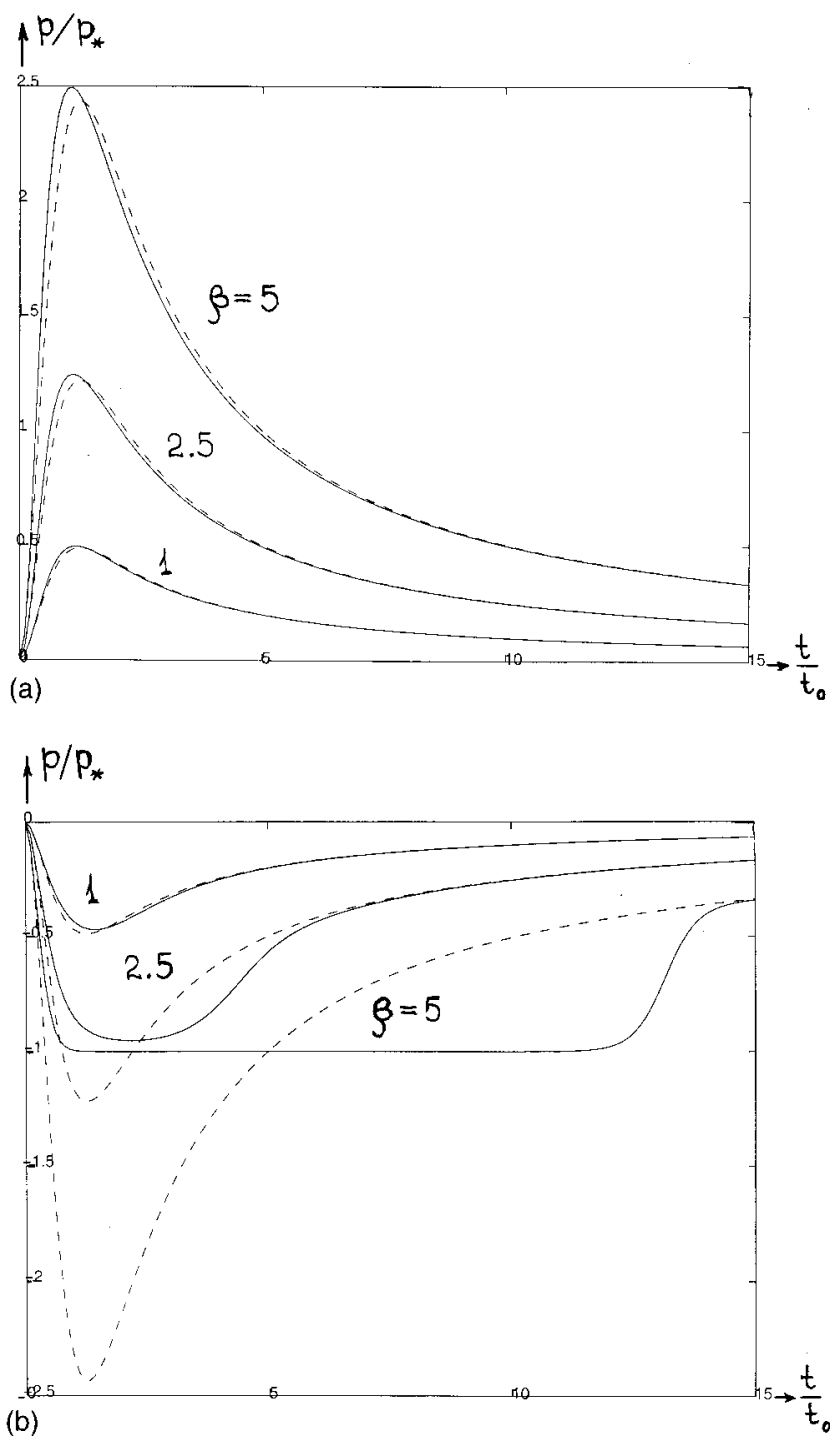

FIG. 8. The same as in Fig. 7, but for $a=5$.

The extremal peak pressure (74) can be recorded at the excitation of a layer by a short acoustic pulse. After several $(N)$ measurements, corresponding to different $\left(p_{0}\right)_{n}, n$ $=1,2,3, \ldots, N$, a set of data on peak pressure $\left(p_{\text {ext }}\right)_{n}$ can be formed. Consequently, the relationship (74) can be reconstructed with an accuracy depending on the experimental error. But, the dependence (74) is nothing more or less than the dependence $f(p)=a p_{0}(p)$ used at the derivation of the solution (73). Consequently, this scheme of measurement gives the possibility to evaluate the stress-strain relationship.

A more general scheme follows when considering (70) with its right-hand side corresponding to an arbitrary shape of incident pulse $p_{i}(\tau)$. It is then convenient to write (70) in the form

$$
a\left(p_{i}-p\right) \frac{d p}{d f}=\frac{d p}{d \tau} .
$$

Equation (75) is fulfilled at any moment of $\tau$. Consequently, the derivative $d p / d f$ can be considered as a constant, independent of $\tau$. Multiplying (75) by $p$, it is easy to derive the formula $\left\langle p p_{i}\right\rangle=\left\langle p^{2}\right\rangle$, where the brackets denote the integral of $\tau$ over the region where both $p$ and $p_{i}$ are nonzero. There- 


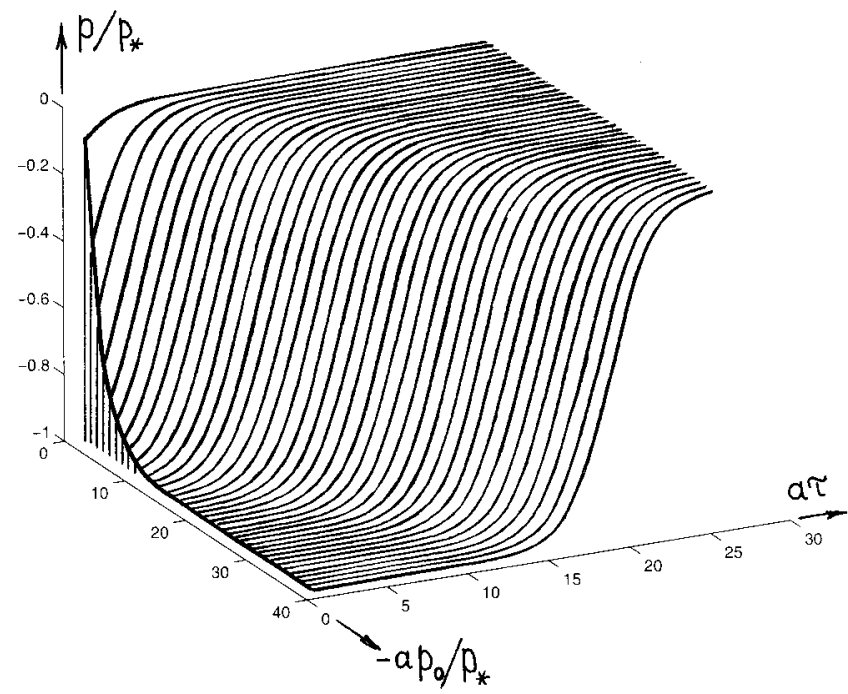

FIG. 9. Strong nonlinear response to a short rarefaction pulse in form of a negative $\delta$ function.

after, multiplying (75) by $p_{i}$ and integrating over $d \tau$ one obtains

$$
a \frac{d p}{d f}=-\frac{\left\langle p d p_{i} / d t\right\rangle}{\left\langle p_{i}^{2}\right\rangle-\left\langle p^{2}\right\rangle} .
$$

Making a series of measurement at different $\left(p_{0}\right)_{n}$, the righthand side $Q$ of (76) can be determined, and (76) takes the form

$$
a\left(\frac{d p}{d f}\right)_{p=\left(p_{0}\right)_{n}}=Q\left[\left(p_{0}\right)_{n}, a\right] .
$$

During measurements carried out in the linear regime (at small $p_{0}$ ) the right-hand side of (76) does not depend on $p_{0}$. This fact makes it possible to determine the constant $a$. Then, at finite and large values of $p_{0}$, after reconstruction of the function $Q$ in a significant number of points, one can integrate (77)

$$
a \frac{d p}{Q(p, a)}=d f,
$$

and restore the stress-strain relationship.

\section{CONCLUSIONS}

Much attention has been given in the last few years to nonlinear evaluation of mechanical properties of materials containing mesoscopic inhomogeneities. There have been organized special conferences and special sessions (see, for example, Refs. 19 and 20) devoted to this problem. A great body of significant experimental data has been obtained, and new mechanical models for nonlinear properties of defective solids were developed.

Remote evaluation and testing includes the processing of acoustic signals scattered by linear or nonlinear inhomogeneities. Consequently, the nonlinear response of different internal defects must be studied.

The statement of the problem to which this paper is devoted is most simple: Find the nonlinear pulse response of a nonresonant layer to a plane incident wave. Because of this simplicity, both weak and strong nonlinear responses were studied in detail. These results can be used in qualitative analyses of more complicated scatterers and structures.

Moreover, this investigation line must be continued. First, the nonlinear responses of inhomogeneities having different shapes, internal structures, and physical properties have to be calculated. Second, the results for single inhomogeneities must be used for calculation of responses from ensembles of such scatterers. Third, direct data can be applied in the solution of inverse problems and nonlinear tomography.

The preliminary results of this work were delivered by Hedberg at the conference ${ }^{20}$ and published as a brief communication. ${ }^{21}$

\section{ACKNOWLEDGMENTS}

Our sincere thanks to P. Johnson, A. Sutin, V. Robsman, I. Solodov, A. E. Sobisevich, and L. E. Sobisevich for fruitful discussions. The work was supported by CRDF, INTAS 9988, and RFBR grants.

${ }^{1}$ K. A. Naugolnykh and L. A. Ostrovsky, Nonlinear Wave Processes in Acoustics (Cambridge University Press, New York, 1998).

${ }^{2}$ O. A. Druzhinin, L. A. Ostrovsky, and A. Prosperetti, "Low-frequency acoustic wave generation in a resonant bubble-layer," J. Acoust. Soc. Am. 100, 3570-3580 (1996).

${ }^{3}$ L. A. Ostrovsky, A. M. Sutin, I. A. Soutova, A. I. Matveyev, and A. I. Potapov, "Nonlinear, low-frequency sound generation in a bubbly layer: Theory and laboratory experiment," J. Acoust. Soc. Am. 104, 722-726 (1998).

${ }^{4}$ B. B. Goldberg, J. B. Liu, and F. Forsberg, "Ultrasound contrast agents: A review," Ultrasound Med. Biol. 20, 4 (1994).

${ }^{5}$ I. Shkolnik, L. Zarembo, and V. Krasilnikov, "On structural nonlinear diagnostics of solids and estimation of their strength," in Frontiers of Nonlinear Acoustics, edited by M. F. Hamilton and D. T. Blackstock (Elsevier, London, 1990), pp. 589-594.

${ }^{6}$ V. A. Robsman and O. V. Rudenko, "A new method for nonlinear nondestructive testing of developing defects inside inhomogeneous solids," J. Acoust. Soc. Am. 105, 1015A (1999).

${ }^{7}$ V. A. Robsman, "Nonlinear transformation of noise spectra in the acoustic diagnostics of concrete structures," Sov. Phys. Acoust. 37, No. 5, 541-543 (1991). "Accumulation and chaotic development of nonlinear acoustical processes in the dynamic loading of geological structures," Acoust. Phys. 39, 176-183 (1993).

${ }^{8}$ T. Sato, "Industrial and medical application of nonlinear acoustics," in Frontiers of Nonlinear Acoustics, edited by M. F. Hamilton and D. T. Blackstock (Elsevier, London, 1990), pp. 98-112.

${ }^{9}$ O. V. Rudenko, "Nonlinear methods in acoustic diagnostics (Review)," Russ. J. Nondestruct. Test. 29, 583-588 (1993).

${ }^{10}$ R. A. Guyer and P. A. Johnson, "Nonlinear mesoscopic elasticity: Evidence for a new class of materials (Review)," in Physics Today, April 1999, pp. 30-36.

${ }^{11}$ K. E-A. Van Den Abeele, P. J. Johnson, R. A. Guyer, and K. R. McCall, "On quasi-analytic treatment of hysteretic nonlinear response in elastic wave propagation,” J. Acoust. Soc. Am. 101, 1885-1898 (1997).

${ }^{12}$ Y. Zheng, R. G. Maev, and I. Yu. Solodov, "Nonlinear acoustic applications for material characterization: A review," Can. J. Phys. 77, 927-967 (1999).

${ }^{13}$ V. A. Burov, I. E. Gurinovich, O. V. Rudenko, and E. Ya. Tagunov, "Reconstruction of the spatial distribution of the nonlinearity parameter and sound velocity in acoustic nonlinear tomography," Acoust. Phys. 40, 816823 (1994).

${ }^{14}$ L. M. Brekhovskikh, Waves in Layered Media, 2nd ed. (Academic, New York, 1980). L. M. Brekhovskikh and O. A. Godin, Acoustics of Layered Media (Springer, New York, 1998).

${ }^{15}$ O. V. Rudenko, and S. I. Soluyan, Theoretical Foundations of Nonlinear Acoustics (Plenum, Consultants Bureau, New York, 1977).

${ }^{16}$ V. G. Andreev, V. Yu. Veroman, G. A. Denisov, O. V. Rudenko, and O. A. 
Sapozhnikov, "Nonlinear acoustical aspects of extracorporeal lithotripsy," Sov. Phys. Acoust. 38, 325-328 (1992).

${ }^{17}$ I. Yu. Solodov, "Ultrasonics of non-linear contacts: Propagation, reflection, and NDE-applications," Ultrasonics 36, 383-390 (1998).

${ }^{18} \mathrm{O}$. V. Rudenko and C. A. Vu, "Nonlinear acoustic properties of a rough surface contact and acoustodiagnostics of a roughness height distribution," Acoust. Phys. 40, 593-596 (1994).
${ }^{19}$ Nonlinear Acoustics at the Turn of the Millenium, Proceedings of the 15th International Symposium on Nonlinear Acoustics, edited by W. Lauterborn and T. Kurz (AIP, Melville, NY, 2000).

${ }^{20}$ Fifth Annual Workshop on Nonlinear Mesoscopic Elasticity, Santa Margherita Ligure, Italy, 4-9 July 2000.

${ }^{21}$ O. V. Rudenko, L. E. Sobisevich, A. L. Sobisevich, and C. M. Hedberg, "Nonlinear response of a layer to pulse action in diagnostics of small inhomogeneities," Dokl. Phys. 45, 485-488 (2000). 